\title{
UCRL-JRNL-224567
}

LA W REN CE LIVERMORE N A TIONAL LABORATORY

\section{The effect of temporal pulse shape on optical damage}

C. W. Carr, J. B. Trenholme, M. L. Spaeth

September 20, 2006

Applied Physics Letters 
This document was prepared as an account of work sponsored by an agency of the United States Government. Neither the United States Government nor the University of California nor any of their employees, makes any warranty, express or implied, or assumes any legal liability or responsibility for the accuracy, completeness, or usefulness of any information, apparatus, product, or process disclosed, or represents that its use would not infringe privately owned rights. Reference herein to any specific commercial product, process, or service by trade name, trademark, manufacturer, or otherwise, does not necessarily constitute or imply its endorsement, recommendation, or favoring by the United States Government or the University of California. The views and opinions of authors expressed herein do not necessarily state or reflect those of the United States Government or the University of California, and shall not be used for advertising or product endorsement purposes. 


\title{
The effect of temporal pulse shape on optical damage
}

\author{
C.W. Carr, J.B. Trenholme and M. L. Spaeth \\ Lawrence Livermore National Laboratory, 7000 East Ave., Livermore, CA 94550
}

\begin{abstract}
(Received)
The conditions under which optical materials are susceptible to laser-induced damage is a topic which has been the subject of considerable study. Laser parameters such as wavelength and temporal pulse duration have been studied extensively. Until this work the effect of temporal pulse shape has not been considered. We present here data from a simple single-parameter model and a supporting experiment which predicts that a Flat-InTime-pulse will produce damage at approximately $80 \%$ of the fluence of a Gaussian pulse of the same FWHM duration. (c) 2006 TBD OCIS codes: $140.3330,160.4330, \ldots$
\end{abstract}

The most cost-effective laser design for both large-aperture and table-top lasers requires optical components to be operated at energy densities (fluences) near that those which will cause damage to the system. Several laser parameters have a significant effect on the maximum safe operating fluence of a laser system. These include wavelength, pulse duration, beam contrast, and temporal pulse shape [1-5]. These coupled laser parameters define a huge parameter space, making testing at every combination problematic. It is therefore highly desirable to understand the effect of each parameter individually. As an example, for third harmonic light (355 nm) the effect of pulse duration and beam contrast is predictable for pulse durations between 1 nanosecond and 30 nanoseconds. However, no method for evaluating the effect of pulse shape yet exists within the literature. The effect of the complex temporal shape of the pulses used in large fusion-research laser systems is of critical importance. In this work we present and verify an empirical model to predict the effect on damage initiation as a function of temporal pulse shape.

Because damage data has historically been taken with pulses whose temporal shapes were (more or less) Gaussian[2, 6-10], it is desirable to base our predictions on data taken with Gaussian pulses of different pulse widths. To do so, we must adopt some parametric model of the damage process. We will determine the model parameters for an arbitrary material by fitting the model to the Gaussian-pulse data. We will then apply the model to the particular case of damage in the bulk of DKDP crystals.

Experimentally, the fluence required to cause a damage initiation density of a few sites per square centimeter or cubic millimeter (for surface or volume damage, respectively) is found to increase as a power of the pulse duration for pulse widths from 10 ps to $300 \mathrm{~ns}$, for a range of wavelengths, in a number of different materials[5, 11, 12] This very broad pulse-width range of power-law dependence is quite remarkable, since most physical characteristics are not ruled by one process over such a large variation of a single parameter. The measured value of the power varies with wavelength and material, and usually lies between 0.15 and 0.5. In general, the initiation fluence is given by

$$
\Phi_{I}(W)=C W^{\beta}
$$

Here $\beta$ is the power, $W$ is the pulse width (typically the FWHM of a Gaussian) and C is a constant.

Simple diffusion from one-dimensional (plate-like), twodimensional (rod-like) or three-dimensional (ball-like) source regions cannot explain the observed power-law dependence. Therefore we shall adopt a phenomenological model. It is approximately diffusive, although it does not correspond to any simple diffusion picture. We assume that damage is caused by the accumulation of a substance D we refer to as "damageonium.” This substance is most probably heat, but it might be electrons, or some other quantity that causes damage if it exceeds a local density limit $\mathrm{D}_{\mathrm{I}}$. We assume that $\mathrm{D}$ is produced when laser light is linearly absorbed in the volume of small (sub-wavelength) damage precursor structures of some kind, and that it diffuses away from the volume of production. This causes the density of $\mathrm{D}$ to rise near the absorption volume, but because of the diffusion the rise is not linearly proportional to the energy density per unit area (fluence) in the pulse.

We also assume that once the density of $\mathrm{D}$ rises above the threshold $\mathrm{D}_{\mathrm{I}}$, a runaway process takes place and the local absorption rises sharply, leading to damage initiation. This means that we are ignoring the possibility of damage due to the effects of a quantity such as stress which effectively integrates D over a volume, and which therefore sees some spatial average of $\mathrm{D}$. The spatial and temporal peak of $\mathrm{D}$ is what matters.

We presume that there are very many absorption sites within the laser beam footprint, on the surface or in the bulk of a material, which is generally the cases for spot sizes larger 
than a few $\mathrm{mm}$ in diameter[13, 14], and that the damage initiation density at any specific fluence measures the number of precursors that have undergone runaway[15].

Recall that the Green's function for delta-function deposition in space and time of a diffuser in an infinite uniform isotropic medium is

$$
g(t, s)=\frac{e^{\frac{-s^{2}}{4 \kappa t}}}{(4 \pi \kappa t)^{d / 2}}
$$

Here ' $\mathrm{s}$ ' is the distance from the deposition point, ' $\mathrm{t}$ ' is the elapsed time since deposition, and ' $K$ ' is the medium's diffusion constant. The dimensionality of the diffusion region is given by 'd,' with $\mathrm{d}=1$ corresponding to plate-like $1 \mathrm{D}$ diffusion, $d=2$ to rod-like 2D diffusion, and $d=3$ to ball-like 3D diffusion. We use only the power-law behavior of the denominator in the Green's function in our model (that is, we presume the maximum $\mathrm{D}$ value is at the deposition point), and presume that the temporal behavior of $\mathrm{D}$ is given by the convolution integral

$$
D(t)=A \int_{0}^{\infty} \frac{\left(1-\frac{d}{2}\right) P(t-u)}{u^{d / 2}} d u
$$

The constant A depends on the physics and geometry. It will drop out later when we take ratios. $\mathrm{P}(\mathrm{t})$ is the temporal shape of the laser pulse. The factor of $(1-\mathrm{d} / 2)$ is just for mathematical convenience, and will also drop out when we take ratios. The model depends only on the single parameter $\mathrm{d}$. The convolution integral has an integrable singularity for very short delay times from deposition. Presumably the actual behavior at such times corresponds to a linear temperature rise with no diffusion, and we should modify the convolution kernel to correspond to such behavior (leaving the area under the kernel for short times unchanged), but this will only change the behavior of the model for pulse changes over times comparable to 10 ps or so. We therefore keep the simpler form used above.

We use the dimension parameter ' $d$ ' to fit experiment. It is easily seen that damage in this model scales as pulse duration to the power $d / 2$. We therefore equate $\beta$ and $d / 2$. For the observed values of $\beta=0.15$ to $\beta=0.5$, we see that a dimension of $d=0.3$ to $d=1$ is implied. Thus the model involves diffusion in "fractional dimensions." Such diffusion cannot take place in simple geometries, but fractional dimensionality is the norm in fractal structures, where detail repeats at different scales. Perhaps the observed damage behavior is due to multi-scale structure at the 1 to $100 \mathrm{~nm}$ size, such as complicated multi-fracture zones or nano-voids with complex internal morphologies.

We have assumed that the absorption and diffusion are independent of temperature, leading to a linear convolution integral. Dimensional arguments provide by D. Eimerl in 1994 show that the pulse-width scaling is unchanged if either or both are dependent on temperature, so we do not need to consider this possibility.

We now find the Gaussian pulse that will initiate damage at a fluence of $C W^{\beta}$. The pulse has the shape

$$
P_{I}(t)=\frac{2 C \sqrt{\ln (2)} e^{\frac{-4 t^{2} \ln (2)}{W^{2}}}}{W^{1-\beta} \sqrt{\pi}}
$$

Recall that $\mathrm{W}$ is the FWHM of the Gaussian. Normalizing the convolution integral we find the temporal behavior of $\mathrm{D}$ that just leads to damage initiation to be the maximum over $t$ of

$$
D_{I}(t)=2 A C \sqrt{\frac{\ln (2)}{\pi}}(1-\beta) \int_{0}^{\infty} \frac{e^{-4(r-s)^{2} \ln (2)}}{s^{\beta}} d s
$$

Here ' $r$ ' is the normalized time $r=t / W$.

To find the maximum value of $\mathrm{D}$ for Gaussians, we want the maximum of the normalized portion of this over $t$, with $\beta$ as a parameter. Call the maximum value $K(\beta)$, so that

$$
\begin{aligned}
& \mathrm{K}(\beta)={ }_{r}^{\max }\left[(1-\beta) \int_{0}^{\infty} \frac{e^{-4(r-s)^{2} \ln (2)}}{s^{\beta}} d s\right] \\
& \mathrm{K}(0)=\sqrt{\frac{\pi}{4 \ln (2)}} \\
& \mathrm{K}(1)=1
\end{aligned}
$$

There is an analytic form for the integral in terms of Laguerre polynomials of fractional order, but it is easier to find the maxima numerically. The resulting numeric values can then be approximated by a Padé form. We find that $K(\beta)$ is approximated to $20 \mathrm{ppm}$ over the range $\beta=0.005$ to $\beta=1$ by the expression

$K(\beta) \cong \frac{1.06+\sqrt{\beta}(0.751-\sqrt{\beta}(1.64-\sqrt{\beta} 0.920))}{1+\sqrt{\beta}(0.712-\sqrt{\beta}(0.121-\sqrt{\beta}(0.00732-\sqrt{\beta} 0.503)))}$

We now find the damage initiation fluence for any arbitrary pulse shape by equating the initiating $\mathrm{D}$ level for that pulse to the initiating level for a Gaussian pulse. Write the pulse shape of the arbitrary pulse as

$$
P(t)=P_{M A X} n(t)
$$

where $P_{\mathrm{MAX}}$ is the peak power in the pulse and $n(t)$ is the pulse shape normalized to unit peak value.

To get our result, we need the energy-equivalent Gaussian pulse width. This is the FWHM of a Gaussian pulse that has the same peak power, and same energy, as the arbitrary pulse. It is given by

$$
W_{G E}=\frac{2 \Phi}{P_{M A X}} \sqrt{\frac{\ln (2)}{\pi}}
$$


Use this to replace $\mathrm{P}_{\text {MAX }}$ in the convolution integral, getting

$$
D(t)=\frac{2 A \Phi}{W_{G E}} \sqrt{\frac{\ln (2)}{\pi}}(1-\beta) \int_{0}^{\infty} \frac{n(t-u)}{u^{\beta}} d u
$$

Equate the peak values of D in the Gaussian convolution and the arbitrary-pulse convolution, and solve for the initiation fluence. Many common factors drop out (as promised) leaving the result

$$
\Phi_{I}=\frac{C W_{G E}^{\beta} K(\beta)}{\max \left[(1-\beta) \int_{0}^{\infty} \frac{n\left(t-W_{G E} s\right)}{s^{\beta}} d s\right]}
$$

Using this result, we can find the pulse width (FWHM) of a Gaussian pulse, having the same energy (or fluence) as the arbitrary pulse, that just initiates damage. This is the "damage-initiation-equivalent Gaussian FWHM," and is given by

$W_{G I}=W_{G E}\left\{\frac{K(\beta)}{\max \left[(1-\beta) \int_{0}^{\infty} \frac{n\left(t-W_{G E} s\right)}{s^{\beta}} d s\right]}\right\}^{\frac{1}{\beta}}$

It is convenient to approximate the arbitrary pulse by a sequence of piecewise-linear sections, since the integral is easily done for such segments. The piecewise-linear results are then added, and the maximum is found numerically.

In the particular case of a flat-in-time (FIT) or square temporal shape, the energy-equivalent Gaussian pulse width is easily seen to be

$$
W_{G E}=2 \sqrt{\frac{\ln (2)}{\pi} \tau}
$$

where $\tau$ is the pulse duration. The factor in front of $\tau$ is equal to 0.939 . The maximum of $\mathrm{D}$ comes at the end of the pulse, so the damage-initiation-equivalent Gaussian FWHM is readily found to be

$W_{G I}=\tau\left[2 \sqrt{\frac{\ln (2)}{\pi}} K(\beta)\right]^{\frac{1}{\beta}}$

To test this model we now apply it to bulk damage initiated with a temporally Gaussian and a temporally flat-in-time (FIT) pulses in a DKDP crystal. A large body of work exploring the damage behavior of bulk damage behavior exists[1, 2, 9, 16-22], but for the purpose of testing the model we need only state that for damage initiated with third harmonic light at high fluence? precursors are known to be present in excess of $10^{4}$ per $\mathrm{mm}^{3}$ and that the measured pulse scaling is $\sim \mathrm{t}^{0.35+/-0.05} \cdot[20,23]$

The experimental details of the damage measurement are described in detail elsewhere (MST). Four 1-cm thick DKDP samples, each cut from a different boule, had two nonoverlapping but adjacent volumes exposed to $\sim 1$-cm diameter laser pulses with FIT and Gaussian temporal profiles (an example of which may be seen in the inset of figure 1) with $\sim 3$-ns full-width at half maximum intensities. All eight laser pulses had a whole-beam mean fluence of approximately 7 $\mathrm{J} / \mathrm{cm}^{2}$. The mean fluence of the pulses in the figure 1 inset were $6.7 \mathrm{~J} / \mathrm{cm}^{2}$ and $6.1 \mathrm{~J} / \mathrm{cm}^{2}$ for the Gaussian and FIT pulses, respectively. The local fluence of the pulses varied spatially and therefore produced damage within the beam footprint with the same spatial variations. The local density of damage sites was measured with an automated microscope and then correlated to the local fluence which produced it. A plot of density of damage sites vs fluence for the FIT and Gaussian pulses from the inset may be seen figure 1 .

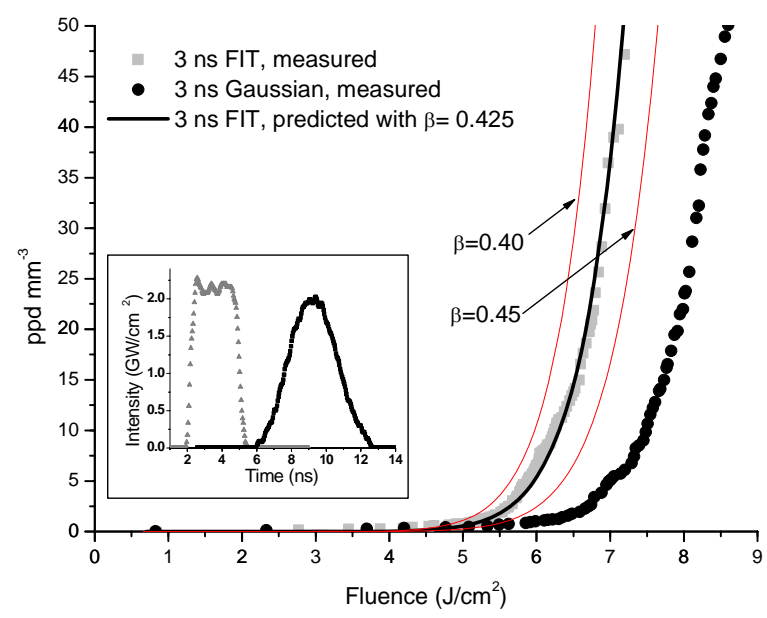

Figure 1. Measured density vs fluence curves for 3-ns FIT and Gaussian pulses and the density vs fluence curve of a 3-ns FIT pulse predicted from the 3-ns Gaussian pulse data with $\beta=0.425$ and nearby values. The inset depicts the temporal trace of both pulses.

For fluences above the onset of observable damage the density of damage for a given fluence is seen to differ significantly for FIT and Gaussian pulses. The measured data in Fig. 1 as well as the data from the other four samples (see Table 1) indicate that the fluence needed to cause observable damage with a FIT pulse of the same FWHM duration is $80 \pm$ $5 \%$ of that needed for a Gaussian pulse.

\begin{tabular}{|l|l|l|}
\hline Sample & $\phi_{\mathrm{FIT}} / \phi_{\text {Gaussian }}$ & $\beta$ \\
\hline $\mathrm{A}$ & $83 \%$ & 0.4 \\
\hline $\mathrm{B} *$ & $85 \%$ & 0.425 \\
\hline $\mathrm{C}$ & $80 \%$ & 0.37 \\
\hline $\mathrm{D}$ & $78 \%$ & 0.35 \\
\hline
\end{tabular}

Table 1 . The percentage difference in fluence $(\phi)$ for observable damage by a FIT vs Gaussian pulse. *The Pulse shapes and damage vs density curves from sample B are shown in figure 1.

To apply the model we must adjust our free parameter $(\beta)$ in equation 5 to get the experimentally observed pulse-width dependence. From the model, the ratio of fluences needed to 
produce the same damage level with FIT and Gaussian pulses of the same FWHM is

$\frac{\Phi_{F I T}}{\Phi_{G}}=\frac{C W_{G E}^{\beta} K(\beta)\left(\frac{W_{G E}}{\tau}\right)^{1-\beta}}{C W_{G}^{\beta}}=2 \sqrt{\frac{\ln (2)}{\pi}} K(\beta)$

Using the $\beta=0.37$ from pulse duration experiments (runkel), we have $\mathrm{K}(\beta)=0.849$ and the fluence ratio becomes 0.797. This matches sample C (table 1). Other similar values match the other samples in table 1 . The average $\beta$ for the four samples is 0.39 . Thus the single-parameter diffusive model presented here accurately predicts the effect of a pulse shape change from Gaussian to flat-in-time with an average value for $\beta$ of 0.39 (dimensionality of 0.78 ). This is consistent with the results of Runkel et al. for pulse scaling with Gaussian pulses of different FWHM. The effect of the complex temporal shape can be predicted in the same fashion as used for different pulse durations of Gaussian pulses.

\section{Acknowledgements}

Worked performed under the auspices of the U.S. Department of Energy by the University of California, Lawrence Livermore National Laboratory under contract No. W-7405-Eng-48.

[1] C. W. Carr, H. B. Radousky, and S. G. Demos, Phys Rev Lett 91, 127402 (2003).

[2] H. Yoshida et al., Appl Phys B-Lasers Opt 70, 195 (2000).

[3] M. Runkel et al., in Boulder Damage Symposium (SPIE, Boulder, CO, 2000), p. 359.
[4] P. DeMange et al., Optics Letters 30, 221 (2005).

[5] J. Campbell, and F. Rainer, (SPIE, 1992), p. 246.

[6] M. Hercher, J. Opt. Soc. Am. (USA) 54, 536 (1964).

[7] N. Bloembergen, IEEE J. Quantum Electron. (USA) QE-10, 375 (1974).

[8] J. Swain et al., Applied Optics Letters 40, 350 (1982).

[9] F. Rainer, L. J. Atherton, and J. J. D. Yoreo, in Laser-Induced Damage in Optical Materials: 1992 (SPIE, Boulder Co., 1992), p. 46.

[10] J. J. Adams et al., (SPIE, 2004), p. 265.

[11] D. Cross, and C. W. Carr, TBD (In preperation).

[12] J. J. Adams et al., in Laser-Induced Damage in Optical Materials: 2005, edited by G. J. Exarhos et al. (SPIE, Boulder, CO, 2005), p. 220.

[13] P. DeMange et al., Review of Scientific Instruments 75, 3298 (2004).

[14] C. W. Carr et al., Measurement Science and Technology (2006).

[15] C. W. Carr, and J. M. Arabach, Optics Letters (2006).

[16] J. Swain et al., Appl Phys Lett 40, 350 (1982).

[17] A. Yokotani et al., Appl Phys Lett 48, 1030 (1986).

[18] E. M. Campbell, Fusion Technol 26, 755 (1994).

[19] M. Runkel, and R. S. III, 1999).

[20] A. K. Burnham et al., Appl. Opt. (USA) 42, 5483

(2003).

[21] C. W. Carr et al., Optics Letters 30, 661 (2005).

[22] C. W. Carr et al., Phys Rev Lett 92, 087401 (2004).

[23] M. Runkel et al., in Boulder Damage Symposyem

(SPIE, Boulder, CO, 2001), p. 359. 